\title{
Invited Article
}

\section{Anterior craniofacial resection - for paranasal sinus tumors involving anterior skull base}

\author{
Saurabh Varshney · S. S. Bist · Nitin Gupta · R. K. Singh - Sanjeev Bhagat
}

\begin{abstract}
Management of anterior skull base tumors is complex due to the anatomic detail of the region and the variety of tumors that occur in this area. Currently, the "gold standard" for surgery is the anterior craniofacial approach. Craniofacial resection represents a major advance in the surgical treatment of tumors of the paranasal sinuses involving anterior skull base. It allows wide exposure of the complex anatomical structures at the base of skull permitting monobloc tumor resection. This study presents a series of 18 patients with anterior skull base tumors, treated by a team of head-neck surgeons and neurosurgeons. The series included 15 malignant tumors of the nose and paranasal sinuses and 3 extensive benign lesions. All tumors were resected by a combined bi-frontal craniotomy and rhinotomy. The skull base was closed with a pediculated pericranial flap and a split-thickness free skin graft underneath. There were no postoperative problems of wound infection, cerebrospinal fluid-leakage or meningitis. Recurrent tumor growth or systemic metastasis occurred in 3 out of 15 patients with malignant tumors, 6 months to 2 years postoperatively. Craniofacial resection was thus found to give excellent results with low morbidity in malignant lesions and can also be adapted for benign tumors of anterior skull base.
\end{abstract}

Keywords Craniofacial approach · Anterior skull base tumor

\section{Background}

In 1954, Smith reported the first anterior craniofacial resection in a patient presenting with a tumor arising in the frontal sinus. Ketcham et al. reported the first series of patients treated with an anterior craniofacial resection for tumors arising in the ethmoid sinuses. This report included the indications, morbidity, and outcome of the procedure and included a systematic description of the surgical technique. The oncologic principles of anterior craniofacial resection remain as described by Ketcham and involve an enbloc resection of tumor, including the ethmoid sinuses, superior nasal septum, and floor of the anterior cranial fossa, corresponding to the interorbital area (i.e. anterior craniofacial resection) or extended laterally to include part of the bony orbit or its soft tissue contents (anterolateral craniofacial resection). Subsequent reports included larger series of patients and multiple modifications of Ketcham's original surgical technique. These latter reports incorporate technical advances brought to craniofacial surgery by Tessier, such as the use of the subfrontal technique adopted for oncologic surgery by Derome. These modifications improved the visualization of the tumor, facilitating its total removal, and decreased, although did not eliminate, the morbidity due to brain retraction. Recently, endoscopic and endoscopic-assisted techniques have been developed to extirpate selected tumors that traditionally have been resected using a subfrontal approach.

\section{Introduction}

Malignant tumors of the sinonasal tract comprise approximately $3 \%$ of the malignancies that arise in the upper aerodigestive tract. Approximately $10 \%$ of tumors that arise in the sinonasal tract originate in the ethmoid and/or frontal sinuses and are likely to involve the anterior cranial base. The craniofacial approach has gradually evolved over the past five decades into a safe and reliable technique for
S. Varshney $\cdot$ S. S. Bist $\cdot$ N. Gupta $\cdot$ R. K. Singh $\cdot$ S. Bhagat Department of ENT and Head Neck Surgery, Himalayan Institute of Medical Sciences (HIHT University), Jollygrant, Doiwala, Dehradun - 248140 (Uttaranchal), India

S. Varshney $(\bowtie)$

E-mail: drsaurabh68@gmail.com 
resecting both benign and malignant tumors involving the anterior cranial base. It can be extended and incorporated as a part of a more complex resection involving the infratemporal fossa and anterolateral cranial base, as well as the middle cranial fossa, cavernous sinus, etc. The tumors most commonly requiring combined anterior craniofacial surgery usually begin in the nose or sinuses. Tumors with a primary intracranial origin such as meningioma, chordoma or chondrosarcoma require combined resection when they clearly violate the anterior fossa floor.

Cancers of the paranasal sinuses or nasal cavity are the most common malignant tumors of the anterior skull base. A wide variety of tumors occur in this location, such as cancers of endodermal, mesodermal and epidermal origins, including squamous cell carcinoma, melanoma, lymphoma, sarcoma, hemangiopericytoma, malignant giant cell tumor, basal cell carcinoma, plasmacytoma, adenoid cystic carcinoma, mucoepidermoid carcinoma, malignant meningioma and metastatic malignancies.

\section{Surgical technique}

The anterior craniofacial approach incorporates a combination of transfacial and transcranial procedures. The facial approach consists of a graduated greater exposure depending on the extent of disease. The basic is done through a lateral rhinotomy approach coupled with a low craniotomy. The lateral rhinotomy incision may be extended into a Weber-Ferguson incision if a more extensive maxillary excision is required. If preoperative imaging studies confirm the presence of tumor in orbit, the soft tissues of the orbit, then orbital exenteration may be facilitated by extending the incision laterally to include a portion of the eyelids.

The craniotomy is tailored according to the extent of involvement of the anterior fossa floor, the subcranial tumor location, and the degree of dural or frontal lobe invasion. A bi-coronal scalp incision is made running $2-3 \mathrm{~cm}$ behind the hairline. The flap is elevated in the subgaleal plane down to the eyebrows, then to the lateral orbital walls laterally and just below the nasal glabella medially. A large flap of pericranial tissue is created that will be used for later reconstruction. As the dissection proceeds the brows, the supratrochlear and supraorbital neurovascular bundles are exposed and preserved.

The anterior cranial fossa is then exposed by removing a segment of bone which may be pedicled on the temporalis muscle or completely separated. The lower horizontal bone cut should be kept low to lessen the need for subsequent brain retraction. Withdrawing $25-50 \mathrm{ml}$ of cerebrospinal fluid from the lumbar subarachnoid catheter, lowering $\mathrm{PcO}_{2}$ through controlled hyperventilation, and occasionally administering mannitol or steroids further reduce the need for mechanical frontal lobe retraction.
The dura is then carefully dissected off the cristagalli and cribriform plate dividing the dural sleeves that extend along the olfactory nerves. The intracranial portion of the tumor extension is then assessed. If it involves the dura or in certain situations, frontal lobe this will have to be resected, together with the tumor, if the dura is intact, it is retracted back to the planum sphenoidale.

Once the head and neck surgeon has completed the exposure and mobilization of the tumor transfacially a chisel or drill is used either from above or below to make the necessary bone cuts to encompass the tumor and deliver the specimen (Fig. 1 and 4).

\section{Reconstruction}

The pericranium is used for anterior cranial fossa reconstruction. Unless a large amount of anterior cranial fossa bone has been resected and concern for brain herination exists, it is usually not necessary to place a bone graft across the bony defect.

In all cases, an exclusive nasal pack is placed for at least 5 days postoperatively and a lumbar drain kept for the same duration. In significantly larger defects, particularly if orbital exenteration and facial skin is excised, a bulky free flap is considered.

\section{Imaging studies}

Imaging using CT scan and/or MRI is recommended to delineate the extent of the tumor, especially in areas that are not amenable to endoscopic examination, such as the cranial cavity, orbit, other paranasal sinuses, and soft tissues of the face, pterygopalatine, and infratemporal fossa. CT scanning is superior to MRI in defining the bony boundaries. Use of contrast during CT scanning can help estimate the vascularity of the tumor and demonstrates its relationship to the great vessels and other neurovascular structures such as dura, brain and cranial nerves. MRI usually is reserved for patients presenting with invasion of soft tissues, most importantly orbit and brain. Positron

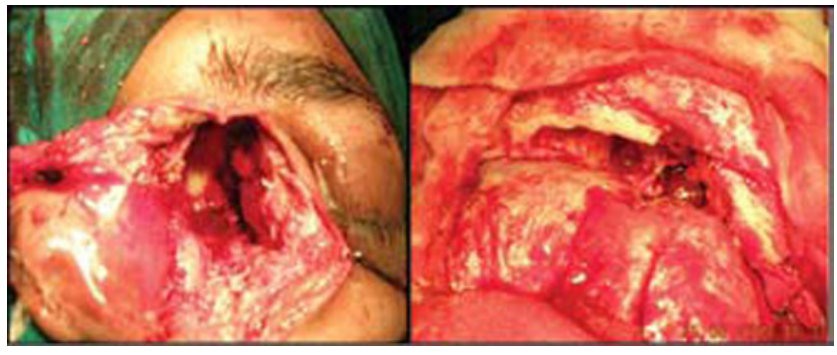

Fig. 1 Lateral rhinotomy (left) and defect (skull base) after tumor removal 
emission tomography (PET) may help to identify the presence of metastatic or recurrent tumor that may escape detection by clinical examination or CT and MRI.

A metastatic workup including CT scan of the chest and abdomen and a bone scan is recommended for patients presenting with tumors that metastasize hematogenously, such as sarcomas, melanomas or adenoid cystic carcinomas.

This study is a series of 18 patients with anterior skull base tumors, treated by a team of head-neck surgeons and neurosurgeons from 2002 to 2008 (Table 1 and 2). The various pathological entities included 15 patients with malignant lesions and 3 with benign pathology. Age of patients ranged from 11 to 62 years (mean age 38.4 years), with male predominance $(\mathrm{M}: \mathrm{F}=3.5: 1.0)$. In all patients a detailed clinical and radiological assessment was made. Axial and coronal CT scans (Figs. 2 and 3) were done to assess extent of the tumor and for planning the surgical procedure, 5 patients had MRI also as radiological investigation, besides diagnostic nasal endoscopy and preoperative biopsy in all. Patients in malignant group with tumor extension into sphenoid sinus, orbital apex infratemporal or pterygopalatine fossa or with brain parenchyrnal involvement were not considered for surgery. Out of 15 malignant tumors, squamous cell carcinoma was the commonest $(22.22 \%)$, followed by adenocarcinoma $(16.67 \%)$ and esthesioneuroblastoma (16.67\%). Orbital invasion was evident radiologically in $3(16.7 \%)$ cases, in which orbital exenteration was also done. Dural invasion was seen on MRI in 2 cases (11.1\%), and they received radiotherapy after surgery. Amongst the 15 patients of malignant tumor a craniofacial resection was combined with total maxillectomy in 5 and a partial maxillectomy in the remaining 10 cases. Orbital exenteration was performed concurrently in 3 cases. The presenting features in benign lesions were proptosis of long duration, diminished vision and headache. Benign lesions predominantly involved the fronto-ethmoid-orbital area (Fig. 3). Most of the tumors had epicenter in nasal cavity $(27.7 \%)$ or ethmoid sinus $(22.22 \%)$. All tumors were resected by a combined bi-frontal craniotomy and rhinotomy. The skull base was closed with a pediculated pericranial flap and a split-thickness free skin graft underneath). Hypotensive anesthesia was used in all cases in the present study. Thirteen of 15 patients with malignant neoplasms received radiotherapy after surgery whereas, 2 were treated with surgery alone. There were no postoperative problems of wound infection, cerebrospinal fluid-leakage or meningitis. Recurrent tumor growth or systemic metastasis occurred in 3 out of 15 patients with malignant tumors, $(2$ patients had local recurrence and 1 had distant metastasis), 6 months to 2 years postoperatively. However, 12 patients $(66.67 \%)$ are surviving disease-free for periods ranging from 12 to 60 months.

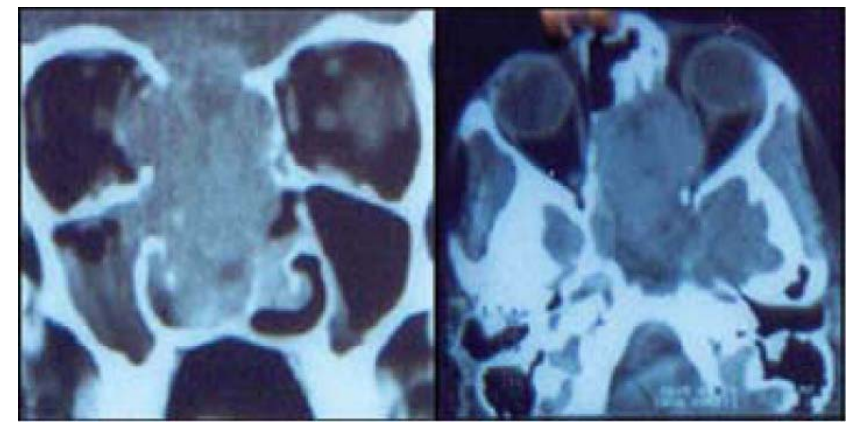

Fig. 2 CT scan coronal/axial view demonstrating a carcinoma of the ethmoid sinuses extending to the orbit and anterior cranial fossa

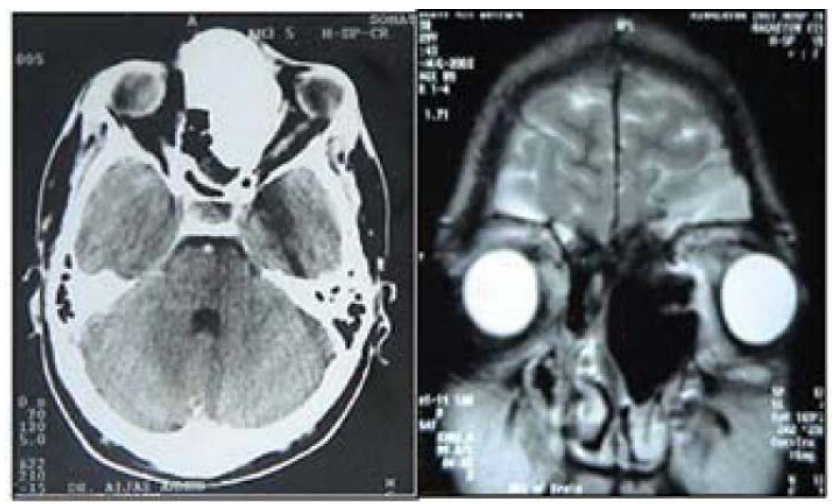

Fig. 3 MRI axial/coronal view demonstrating frontoethmoidal osteoma (presenting as proptosis)

Complications of anterior craniofacial resection

\begin{tabular}{llll}
\hline Wound & Intracranial & Orbital & Endocrine/electrolyte abnormalities \\
\hline Scalp necrosis & Tension pneumocephalus & Epiphora & Hyponatremia (serum sodium $<130 \mathrm{mg} / \mathrm{dl})$ \\
Wound infection & Cerebrospinal fluid leak & Extraocular muscle limitation & Diabetes insipidus \\
Postoperative bleeding & Meningitis/abscess & Enophthalmos & $\begin{array}{l}\text { Hypocalcemia, hypomagnesemia and } \\
\text { hypophosphatemia }\end{array}$
\end{tabular}

Cerebral edema/contusion Blindness 
Table 1 Tumor epicenter

\begin{tabular}{lcc}
\hline Site & No. of cases & Percentage \\
\hline Nasal & 5 & $27.77 \%$ \\
Ethmoid & 4 & $22.22 \%$ \\
Maxilla & 3 & $16.70 \%$ \\
Skin & 1 & $5.54 \%$ \\
Frontal & 1 & $5.54 \%$ \\
Lacrimal & 3 & $16.70 \%$ \\
Orbit & 1 & $5.54 \%$ \\
Total & $\mathbf{1 8}$ & $\mathbf{1 0 0 \%}$ \\
\hline
\end{tabular}

Table 2 Pathology

\begin{tabular}{lcc}
\hline Histology & No. of cases & Percentage \\
\hline Malignant & $(15)$ & $(83.35 \%)$ \\
SCC & 4 & $22.22 \%$ \\
Salivary & 2 & $11.11 \%$ \\
Adeno & 3 & $16.70 \%$ \\
Sarcoma & 1 & $5.54 \%$ \\
Muc. melanoma & 1 & $5.54 \%$ \\
Undiff./Anap. & 1 & $5.54 \%$ \\
Esthesio. & 3 & $16.70 \%$ \\
Benign & $(3)$ & $(16.65 \%)$ \\
Osteoma & 2 & $11.11 \%$ \\
Fibrous dysplasia & 1 & $5.54 \%$ \\
Total & $\mathbf{1 8}$ & $\mathbf{1 0 0 \%}$ \\
\hline
\end{tabular}

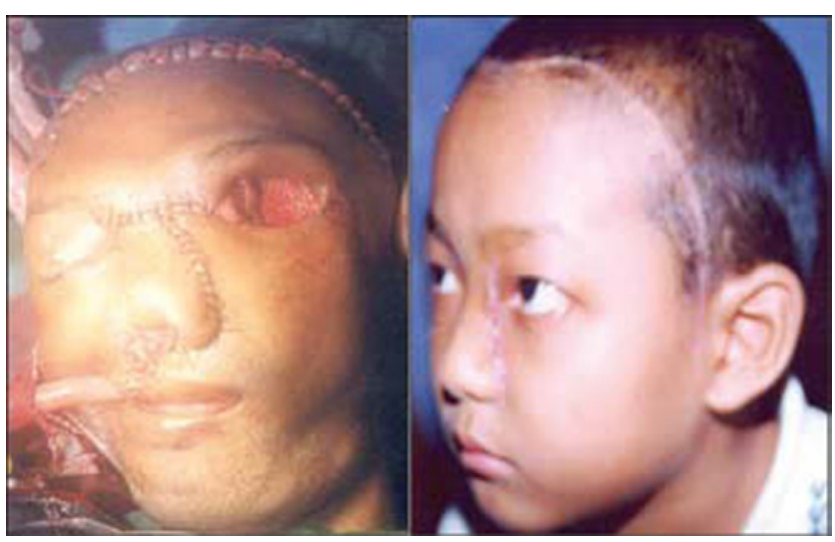

Fig. 4 Markings for a bi-coronal incision, lateral rhinotomy and orbital exentrationa

\section{Future and controversies}

Use of endoscopic techniques to complement or replace traditional approaches is rapidly expanding. Intraoperative navigational devices (computer-assisted surgery) and highdefinition monitors and cameras, customized instruments, and new endovascular neurosurgery techniques that allow intraoperative control of the intracranial vasculature will contribute to the advancement of these techniques. Adjunctive techniques, such as brachytherapy, radiosurgery, intra-arterial chemotherapy and chemotherapy combined with radiation, may have a role in treatment of these lesions. However, the role of these treatments remains undefined. Reports are mainly anecdotal, and their use should be limited to controlled protocols, palliative cases, or poor surgical candidates for whom conventional therapy has failed.

\section{Suggested readings}

1. Blacklock JB, Weber RS, Lee YY, Goepfert H (1989) Transcranial resection of tumors of the paranasal sinuses and nasal cavity. J Neurosurg 71(1):10-15

2. Lund VJ, Howard D, Wei W, Spittle M (2003) Olfactory neuroblastoma: past, present, and future? Laryngoscope 113(3):502-507

3. Levine PA, Debo RF, Meredith SD, Jane JA, Constable WC, Cantrell RW ( ) Craniofacial resection at the University of Virginia (1976-1992): survival analysis. Head Neck 16(6):574-577

4. Lund VJ, Harrison FD (1988) Craniofacial resection for tumors of the nasal cavity and paranasal sinuses. Am J Surg 156(3 Pt 1):187-190

5. Bahadur S, Tandon DA, Mahapatra AK, Misra NK (1996) Craniofacial resection for lesions involving anterior base of skull. Indian J Otolaryngol Head Neck Surg 48(4):295-300

6. Suarez C, Llorente JL, Fernandez de Leon R, Cabanillas R, Suarez V, Lopez A (2004) Anterior craniofacial resection: oncologic outcome and complications in a series of 111 cases. Acta Otorhinolaringol Esp 55(1):27-33

7. Cantu G, Solero CL, Mariani L, Salvatori P, Mattavelli F, Pizzi N, Riggio E (1999) Anterior craniofacial resection for malignant ethmoid tumors - a series of 91 patients. Head Neck 21(3):185-191

8. Goffin J, Fossion E, Plets CH, Mommaerts M, Vrielinck L (1991) Craniofacial resection for anterior skull base tumors. Acta Neurochirurgica 110(1-2):33-37

9. Bridger GP (1980) Radical surgery for ethmoid cancer. Arch Otolaryngol 106(10):630-634

10. Bridger MW, Beale FA, Bryce DP (1978) Carcinoma of the paranasal sinuses - a review of 158 cases. J Otolaryngol 7(5):379-388

11. Cavallo LM, Messina A, Cappabianca P, et al. (2005) Endoscopic endonasal surgery of the midline skull base: anatomical study and clinical considerations. Neurosurg Focus 19(1):E2 
12. Cheesman AD, Lund VJ, Howard DJ (1986) Craniofacial resection for tumors of the nasal cavity and paranasal sinuses. Head Neck Surg 8(6):429-435

13. Clifford P (1980) Transcranial - facial approach for tumors of superior paranasal sinuses and orbit. J R Soc Med 73(6):413-419

14. Eibling DE, Janecka IP, Snyderman CH (1993) Metaanalysis of outcome in anterior skull base resection for squamous cell and undifferentiated carcinoma. Skull Base Surg 3:123-129

15. Myers EN, Carrau RL (1993) Neoplasms of the nose and paranasal sinuses. Head Neck Surg Otolaryngol 1091-1109

16. Patel SG, Singh B, Polluri A, et al. (2003) Craniofacial surgery for malignant skull base tumors: report of an international collaborative study. Cancer 98(6):1179-1187

17. Schramm VL Jr, Myers EN, Maroon JC (1979) Anterior skull base surgery for benign and malignant disease.
Laryngoscope 89(7 Pt 1):1077-1091

18. Shah JP, Sundaresan N, Galicich J, Strong EW (1987) Craniofacial resections for tumors involving the base of the skull. Am J Surg 154(4):352-358

19. Shah JP, Bilsky MH, Patel SG (2002) Malignant tumors of the skull base. Neurosurg Focus 13(4):e6

20. Sisson GA, Bytell DE, Becker SP, Ruge D (1976) Carcinoma of the paranasal sinuses and cranial-facial resection. J Laryngol Otol 90(1):59-68

21. Varshney S, Saxena RK, Bishnu PP, Kaushal A (2004) Sinonasal Haemangiopericytoma. Indian J Otolaryngol Head Neck Surg 56(2):155-158

22. Varshney S, Saxena RK (2004) Adenoid cystic carcinoma nose AND paranasal sinuses. Indian Med Gazet 446-449

23. Saxena RK, Varshney S, Singh J, Kaushal A, Bishnu PP (2001) Haemorrhagic cystic sino-nasal fibrous dysplasia. Indian J Otolaryngol Head Neck Surg 53(2):154-157 